JOURNAL OF THE

CHUNGCHEONG MATHEMATICAL SOCIETY

Volume 27, No. 4, November 2014

http://dx.doi.org/10.14403/jcms.2014.27.4.651

\title{
IMPULSIVE INTEGRAL INEQUALITIES WITH A NON-SEPARABLE KERNEL
}

\author{
Sung Kyu Choi*, Namjip Koo**, and Chunmi Ryu***
}

\begin{abstract}
In this paper we present some Gronwall-tpye inequalities with a non-separable kernel and obtain the explicit estimate for solutions of impulsive differential equations. Furthermore, we give an example to illustrate our results.
\end{abstract}

\section{Introduction}

Integral inequalities of various Gronwall types play important roles in the study of stability of solutions of differential and integral equations, as well as in the modeling of engineering and science problems. Gronwall-type inequalities can be provided explicit bound for solutions of differential and integral equations as well as difference equations. For a detailed discussion of impulsive integral inequalities and some basic concepts concerning about the impulsive differential equations, we refer the reader to $[1,2,4]$.

Differential equations with impulse effect describe evolution process which at certain moments change their state rapidly. In the mathematical simulation of such processes it is convenient to assume that this change takes place momentarily and the process changes its state by jump. Thus, the impulsive differential equations are adequate mathematical models for description of evolution processes characterized by the combination of a continuous and jump change of their states. The theory of impulsive differential equations has found its extensive applications in realistic mathematical modeling of many real world phenomena.

Choi et al. [3] studied $h$-stability for linear impulsive equations using the notion of $t_{\infty}$-similarity and an impulsive integral inequality.

Received August 28, 2014; Accepted September 26, 2014.

2010 Mathematics Subject Classification: Primary 26D15, 34A37.

Key words and phrases: Voltera integral inequality, impulsive integral inequality.

Correspondence should be addressed to Namjip Koo, njkoo@cnu.ac.kr.

This work was supported by research fund of Chungnam National University. 
In this paper we present some Gronwall-tpye inequalities with a nonseparable kernel and obtain the explicit estimate for solutions of impulsive differential equations. Furthermore, we give an example to illustrate our results.

\section{Main results}

Let $P C\left(\mathbb{R}_{+}, \mathbb{R}\right)$ denote the class of piecewise continuous functions with discontinuities of the first kind only at $t=\tau_{k}, k \in \mathbb{N}$.

We need the following conditions:

$\left(H_{1}\right)$ the sequence $\left\{\tau_{k}\right\}$ satisfies $0 \leq t_{0}<\tau_{1}<\tau_{2}<\cdots$, and $\lim _{k \rightarrow \infty} \tau_{k}=$ $\infty$;

$\left(H_{2}\right) m \in P C\left(\mathbb{R}_{+}, \mathbb{R}\right)$ and $m(t)$ is left-continuous at $\tau_{k}, k \in \mathbb{N}$.

We need the Gronwall-type integral inequality with a non-separable kernel to prove our results.

Lemma 2.1. [5, Theorem 1.1.4] Suppose that $h, m \in C\left[\mathbb{R}_{+}, \mathbb{R}_{+}\right], K \in$ $C\left[\mathbb{R}_{+}^{2}, \mathbb{R}_{+}\right], K_{t}(t, s)$ exists, is continuous and nonnegative. Let

$$
m(t) \leq h(t)+\int_{t_{0}}^{t} K(t, s) m(s) d s, t \geq t_{0} .
$$

Then,

$$
m(t) \leq h(t)+\int_{t_{0}}^{t} B\left(s, t_{0}\right) \exp \left(\int_{s}^{t} A\left(\xi, t_{0}\right) d \xi\right) d s, t \geq t_{0},
$$

where

$$
A\left(t, t_{0}\right)=K(t, t)+\int_{t_{0}}^{t} K_{t}(t, s) d s
$$

and

$$
B\left(t, t_{0}\right)=K(t, t) h(t)+\int_{t_{0}}^{t} K_{t}(t, s) h(s) d s .
$$

If we set $h(t)=c$ in Lemma 2.1, then we obtain the following explicit estimate.

Theorem 2.2. Suppose that $m, b \in C\left(\mathbb{R}_{+}, \mathbb{R}_{+}\right), K \in C\left(\mathbb{R}_{+}^{2}, \mathbb{R}_{+}\right), K_{t}(t, s)$ exists, is nonnegative and continuous and for $t \geq t_{0}$,

$$
m(t) \leq c+\int_{t_{0}}^{t} K(t, s) b(s) m(s) d s,
$$


where $c$ is a nonnegative constant. Then, we have

$$
m(t) \leq c \exp \left[\int_{t_{0}}^{t}\left(K(s, s) b(s)+\int_{t_{0}}^{s} K_{s}(s, \sigma) b(\sigma) d \sigma\right) d s\right], t \geq t_{0} .
$$

Proof. If we set $h(t)=c$ in Lemma 2.1, then we have

$$
\begin{aligned}
m(t) & \leq c+c \int_{t_{0}}^{t} A\left(s, t_{0}\right) e^{\int_{s}^{t} A\left(\sigma, t_{0}\right) d \sigma} d s \\
& =c\left(1+\exp \left[\int_{t_{0}}^{t} A\left(s, t_{0}\right) d s\right] \int_{t_{0}}^{t} A\left(s, t_{0}\right) e^{-\int_{t_{0}}^{s} A\left(\sigma, t_{0}\right) d \sigma} d s\right) \\
& =c\left(1+e^{\int_{t_{0}}^{t} A\left(s, t_{0}\right) d s}\left(1-e^{-\int_{t_{0}}^{t} A\left(s, t_{0}\right) d s}\right)\right) \\
& =c \exp \left(\int_{t_{0}}^{t} A\left(s, t_{0}\right) d s\right), t \geq t_{0},
\end{aligned}
$$

where

$$
A\left(t, t_{0}\right)=K(t, t) b(t)+\int_{t_{0}}^{t} K_{t}(t, s) b(s) d s .
$$

Hence, we obtain

$$
m(t) \leq c \exp \left[\int_{t_{0}}^{t}\left(K(s, s) b(s)+\int_{t_{0}}^{s} K_{s}(s, \sigma) b(\sigma) d \sigma\right) d s\right], t \geq t_{0} .
$$

This completes the proof.

COROllary 2.3. If, in Lemma 2.1, $h$ is assumed to be nondecreasing and positive, then the estimate (2.1) reduces to

$$
m(t) \leq h(t) \exp \left[\int_{t_{0}}^{t}\left(K(s, s)+\int_{t_{0}}^{s} K_{s}(s, \sigma) d \sigma\right) d s\right], t \geq t_{0}
$$

Proof. Setting $w(t)=\frac{m(t)}{h(t)}$, we get

$$
w(t) \leq 1+\int_{t_{0}}^{t} K(t, s) w(s) d s, t \geq t_{0}
$$

Hence, we have

$$
w(t) \leq \exp \left[\int_{t_{0}}^{t}\left(K(s, s)+\int_{t_{0}}^{s} K_{s}(s, \sigma) d \sigma\right) d s\right], t \geq t_{0},
$$

by Theorem 2.2, and which yields (2.2). This completes the proof. 
An impulsive integral inequality with a nonseparable kernel can also be reduced to an impulsive differential inequality if the kernel is differentiable. We need the following impulsive integral inequality of Gronwalltype to prove our main results.

Lemma 2.4. [4, Theorem 1.5.4] Assume that $\left(H_{1}\right)$ and $\left(H_{2}\right)$ hold and $m(t) \geq 0$. Suppose that $h \in P C\left(\mathbb{R}_{+}, \mathbb{R}\right), K \in C\left(\mathbb{R}_{+}^{2}, \mathbb{R}_{+}\right), K_{t}(t, s)$ exists, is nonnegative and continuous and for each $k \in \mathbb{N}, t \geq t_{0}$,

$$
m(t) \leq h(t)+\int_{t_{0}}^{t} K(t, s) m(s) d s+\sum_{t_{0}<t_{k}<t} \beta_{k} m\left(t_{k}\right),
$$

where $\beta_{k} \geq 0$ are constants. Then, for $t \geq t_{0}$,

$$
\begin{aligned}
m(t) \leq & h(t)+\sum_{t_{0}<t_{k}<t}\left(\prod_{t_{k}<t_{j}<t}\left(1+\beta_{j}\right) \exp \left(\int_{t_{k}}^{t} A\left(\sigma, t_{0}\right) d \sigma\right) \beta_{k} h\left(t_{k}\right)\right) \\
& +\int_{t_{0}}^{t} \prod_{s<t_{k}<t}\left(1+\beta_{k}\right) \exp \left(\int_{s}^{t} A\left(\sigma, t_{0}\right) d \sigma\right) B\left(s, t_{0}\right) d s
\end{aligned}
$$

where

$$
\begin{aligned}
& A\left(t, t_{0}\right)=K(t, t)+\int_{t_{0}}^{t} K_{t}(t, s) d s, \\
& B\left(t, t_{0}\right)=K(t, t) h(t)+\int_{t_{0}}^{t} K_{t}(t, s) h(s) d s .
\end{aligned}
$$

If we set $h(t)=c$ in Lemma 2.4, then we obtain the following explicit estimate for impulsive integral inequality with a non-separable kernel.

Theorem 2.5. Assume that $\left(H_{1}\right)$ and $\left(H_{2}\right)$ hold and $m(t) \geq 0$. Suppose that $K \in C\left(\mathbb{R}_{+}^{2}, \mathbb{R}_{+}\right), K_{t}(t, s)$ exists, is nonnegative and continuous and for each $k \in \mathbb{N}, t \geq t_{0}$,

$$
m(t) \leq c+\int_{t_{0}}^{t} K(t, s) m(s) d s+\sum_{t_{0}<t_{k}<t} \beta_{k} m\left(t_{k}\right),
$$

where $c$ and $\beta_{k}$ are nonnegative constants. Then, for $t \geq t_{0}$,

$$
\begin{aligned}
m(t) \leq & c \prod_{i=1}^{k}\left(1+\beta_{i}\right) \exp \left[\int_{t_{0}}^{t}(K(s, s)\right. \\
& \left.\left.+\int_{t_{0}}^{s} K_{s}(s, \sigma) d \sigma\right) d s\right], t \in\left(t_{k}, t_{k+1}\right] .
\end{aligned}
$$


Proof. Let $t \in\left(t_{1}, t_{2}\right]$. Then, it follows from Lemma 2.4 that we have

$$
\begin{array}{rl}
m(t) \leq c & c\left[1+\sum_{t_{0}<t_{1}<t} \prod_{t_{1}<t_{j}<t}\left(1+\beta_{j}\right) e^{\int_{t_{1}}^{t} A\left(s, t_{0}\right) d s} \beta_{1}\right. \\
& \left.+e^{\int_{t_{0}}^{t} A\left(s, t_{0}\right) d s} \int_{t_{0}}^{t} \prod_{s<t_{k}<t}\left(1+\beta_{k}\right) A\left(s, t_{0}\right) e^{-\int_{t_{0}}^{s} A\left(\sigma, t_{0}\right) d \sigma} d s\right] \\
= & c\left[1+e^{\int_{t_{1}}^{t} A\left(s, t_{0}\right) d s} \beta_{1}\right. \\
& +e^{\int_{t_{0}}^{t} A\left(s, t_{0}\right) d s}\left(\int_{t_{0}}^{t_{1}}\left(1+\beta_{1}\right) A\left(s, t_{0}\right) e^{-\int_{t_{0}}^{s} A\left(\sigma, t_{0}\right) d \sigma} d s\right. \\
& \left.\left.+\int_{t_{1}}^{t} A\left(s, t_{0}\right) e^{-\int_{t_{0}}^{s} A\left(\sigma, t_{0}\right) d \sigma} d s\right)\right] \\
= & c\left[1+e^{\int_{t_{1}}^{t} A\left(s, t_{0}\right) d s} \beta_{1}\right. \\
& +e^{\int_{t_{0}}^{t} A\left(s, t_{0}\right) d s}\left(\left(1+\beta_{1}\right)\left(1-e^{-\int_{t_{0}}^{t_{1}} A\left(s, t_{0}\right) d s}\right)\right. \\
& \left.\left.+\left(e^{-\int_{t_{0}}^{t_{1}} A\left(s, t_{0}\right) d s}-e^{-\int_{t_{0}}^{t} A\left(s, t_{0}\right) d s}\right)\right)\right] \\
= & c\left(1+\beta_{1}\right) e^{\int_{t_{0}}^{t} A\left(s, t_{0}\right) d s}, t \in\left(t_{1}, t_{2}\right],
\end{array}
$$

where $A\left(t, t_{0}\right)=K(t, t)+\int_{t_{0}}^{t} K_{t}(t, s) d s$.

Let $t \in\left(t_{2}, t_{3}\right]$. Then, by the same method, we also have

$$
\begin{aligned}
m(t) \leq & c\left[1+\left(1+\beta_{2}\right) e^{\int_{t_{1}}^{t} A\left(s, t_{0}\right) d s} \beta_{1}+e^{\int_{t_{2}}^{t} A\left(s, t_{0}\right) d s} \beta_{2}\right. \\
& +e^{\int_{t_{0}}^{t} A\left(s, t_{0}\right) d s}\left(\sum_{i=0}^{1} \int_{t_{i}}^{t_{i+1}} \prod_{s<t_{k}<t}\left(1+\beta_{k}\right) A\left(s, t_{0}\right) e^{-\int_{t_{0}}^{s} A\left(\sigma, t_{0}\right) d \sigma} d s\right. \\
& \left.\left.+\int_{t_{2}}^{t} \prod_{s<t_{k}<t}\left(1+\beta_{k}\right) A\left(s, t_{0}\right) e^{-\int_{t_{0}}^{s} A\left(\sigma, t_{0}\right) d \sigma} d s\right)\right] \\
= & c\left[1+\left(1+\beta_{2}\right) e^{\int_{t_{1}}^{t} A\left(s, t_{0}\right) d s} \beta_{1}+e^{\int_{t_{2}}^{t} A\left(s, t_{0}\right) d s} \beta_{2}\right. \\
& +e^{\int_{t_{0}}^{t} A\left(s, t_{0}\right) d s}\left(\prod_{i=1}^{2}\left(1+\beta_{i}\right)\left(1-e^{-\int_{t_{0}}^{t_{1}} A\left(s, t_{0}\right) d s}\right)\right. \\
& +\left(1+\beta_{2}\right)\left(e^{-\int_{t_{0}}^{t_{1}} A\left(s, t_{0}\right) d s}-e^{-\int_{t_{0}}^{t_{2}} A\left(s, t_{0}\right) d s}\right) \\
& \left.\left.+\left(e^{-\int_{t_{0}}^{t_{2}} A\left(s, t_{0}\right) d s}-e^{-\int_{t_{0}}^{t} A\left(s, t_{0}\right) d s}\right)\right)\right]
\end{aligned}
$$




$$
=c \prod_{i=1}^{2}\left(1+\beta_{i}\right) \exp \left(\int_{t_{0}}^{t} A\left(s, t_{0}\right) d s\right), t \in\left(t_{2}, t_{3}\right] .
$$

Then it follows from mathematical induction that (2.5) holds for $k \in \mathbb{N}$. This completes the proof.

COROLlary 2.6. If $h$ is nondecreasing and positive in Lemma 2.4, then the estimate $(2.4)$ reduces to

$$
\begin{aligned}
m(t) \leq & h(t) \prod_{i=1}^{k}\left(1+\beta_{i}\right) \exp \left[\int_{t_{0}}^{t}(K(s, s)\right. \\
& \left.\left.+\int_{t_{0}}^{s} K_{s}(s, \sigma) d \sigma\right) d s\right], t \in\left(t_{k}, t_{k+1}\right] .
\end{aligned}
$$

Proof. Setting $w(t)=\frac{m(t)}{h(t)}$, we get

$$
w(t) \leq 1+\int_{t_{0}}^{t} K(t, s) w(s) d s+\sum_{t_{0}<t_{k}<t} \beta_{k} w\left(t_{k}\right), t \geq t_{0} .
$$

Hence, we have

$$
\begin{aligned}
w(t) \leq & \prod_{i=1}^{k}\left(1+\beta_{i}\right) \exp \left[\int_{t_{0}}^{t}(K(s, s)\right. \\
& \left.\left.+\int_{t_{0}}^{s} K_{s}(s, \sigma) d \sigma\right) d s\right], t \in\left(t_{k}, t_{k+1}\right],
\end{aligned}
$$

by Theorem 2.5, and which yields (2.6). This completes the proof.

\section{Example}

In this section we give an example which illustrates our results from the previous section.

EXAmple 3.1. [5, Lemma 8.1] We consider the scalar differential equation with impulse effect

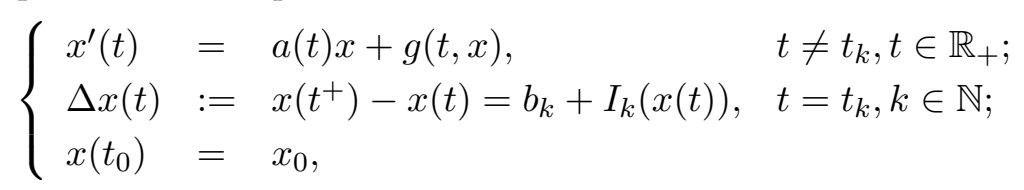

with the following conditions:

(i) The sequence $\left\{t_{k}\right\}$ satisfies $0 \leq t_{0}<t_{1}<t_{2}<\cdots<t_{k}<\cdots$ and $\lim _{k \rightarrow \infty} t_{k}=\infty, k \in \mathbb{N}$. 
(ii) The function $a \in P C\left(\mathbb{R}_{+}, \mathbb{R}\right)$ and $b_{k} \in \mathbb{R}, k \in \mathbb{N}$, and $\prod_{k=1}^{\infty}(1+$ $\left.\left|b_{k}\right|\right)<\infty$.

(iii) Each function $g: \mathbb{R}_{+} \times \mathbb{R} \rightarrow \mathbb{R}$ is continuous and locally Lipschitz continuous with respect to $x$ in the sets $\left(t_{k-1}, t_{k}\right] \times \mathbb{R}, k \in \mathbb{N}$, and for each $k \in \mathbb{N}$ and $y \in \mathbb{R}$, and the limit of $g(t, x)$ exists as $(t, x) \rightarrow\left(t_{k}, y\right), t>t_{k}$. Furthermore, the following inequality holds:

$$
|g(t, x) \leq b(t)| x \mid,
$$

where the function $b \in P C\left(\mathbb{R}_{+}, \mathbb{R}\right)$ is nonnegative.

(iv) The function $I_{k}: \mathbb{R} \rightarrow \mathbb{R}$ is continuous and

$$
\left|I_{k}(x)\right| \leq \rho_{k}|x|, k \in \mathbb{N},
$$

where each $\rho_{k}$ is a nonnegative constant.

(v) $x\left(t_{k}^{+}\right)$represents the right limit of $x(t)$ at $t=t_{k}$.

Together with equation (3.1), we consider the impulsive differential equation

$$
\left\{\begin{array}{l}
x^{\prime}(t)=a(t) x, \quad t \neq t_{k}, t \in \mathbb{R}_{+} ; \\
\Delta x(t)=b_{k}, \quad t=t_{k}, k \in \mathbb{N} ; \\
x\left(t_{0}\right)=x_{0},
\end{array}\right.
$$

and the differential equation

$$
\left\{\begin{array}{l}
x^{\prime}(t)=a(t) x \\
x\left(t_{0}\right)=x_{0} .
\end{array}\right.
$$

Let $u(t, s)$ and $w(t, s)$ be the fundamental solutions of (3.3) and (3.2), respectively. Furthermore, we assume that $u(t, s)$ satisfies the following condition: $|u(t, s)| \leq \varphi(t) \psi(s)$ for $t_{k-1}<s \leq t \leq t_{k}, k \in \mathbb{N}$, where $\varphi, \psi \in$ $P C\left(\mathbb{R}_{+}, \mathbb{R}\right)$ and $\varphi(t)>0, \psi(t)>0, \varphi\left(t_{k}^{+}\right)>0, \psi\left(t_{k}^{+}\right)>0, \varphi\left(t_{k}\right) \psi\left(t_{k}^{+}\right)=1$ for $t \in \mathbb{R}_{+}, k \in \mathbb{N}$. Then the solution $x(t)=x\left(t, t_{0}, x_{0}\right)$ of (3.1) satisfies the following estimate

$$
\begin{aligned}
|x(t)| \leq & c\left|x_{0}\right| \varphi(t) \psi\left(t_{0}^{+}\right) \prod_{t_{0}<t_{k}<t}\left(1+c \rho_{k}\right) \exp \left[\int_{t_{0}}^{t}(b(s)\right. \\
& \left.\left.+\varphi^{\prime}(s) \int_{t_{0}}^{s} \psi(\sigma) b(\sigma) d \sigma\right) d s\right], t \geq t_{0} .
\end{aligned}
$$

Proof. It follows from $[1$, p. 45] that we have

$$
\begin{aligned}
|w(t, s)| & \leq \varphi(t) \psi(s) \prod_{s \leq t_{k}<t}\left(1+\left|b_{k}\right|\right) \varphi\left(t_{k}\right) \psi\left(t_{k}^{+}\right) \\
& \leq c \varphi(t) \psi(s),
\end{aligned}
$$


where $c=\prod_{k=1}^{\infty}\left(1+\left|b_{k}\right|\right)$. In view of the variation in parameters, the solution $x(t)=x\left(t, t_{0}, x_{0}\right)$ of (3.1) satisfies

$$
\begin{aligned}
x(t)= & w\left(t, t_{0}^{+}\right) x_{0}+\int_{t_{0}}^{t} w(t, s) g(s, x(s)) d s \\
& +\sum_{t_{0}<t_{k}<t} w\left(t, t_{k}^{+}\right) I_{k}\left(x\left(t_{k}\right)\right) .
\end{aligned}
$$

From conditions (iii), (iv) and (3.5), we obtain

$$
\begin{aligned}
|x(t)| \leq & c\left|x_{0}\right| \varphi(t) \psi\left(t_{0}^{+}\right)+c \int_{t_{0}}^{t} \varphi(t) \psi(s) b(s)|x(s)| d s \\
& +c \sum_{t_{0}<t_{k}<t} \varphi(t) \psi\left(t_{k}^{+}\right) \rho_{k}\left|x\left(t_{k}^{+}\right)\right|, t \geq s .
\end{aligned}
$$

Setting $m(t)=\frac{|x(t)|}{\varphi(t)}$ and $K(t, s)=\varphi(t) \psi(s) b(s)$, then it follows from Lemma 2.5 that

$$
\begin{aligned}
|x(t)| \leq & c\left|x_{0}\right| \varphi(t) \psi\left(t_{0}^{+}\right) \prod_{t_{0}<t_{k}<t}\left(1+c \rho_{k}\right) \exp \left[\int_{t_{0}}^{t}(b(s)\right. \\
& \left.\left.+\varphi^{\prime}(s) \int_{t_{0}}^{s} \psi(\sigma) b(\sigma) d \sigma\right) d s\right], t \geq t_{0} .
\end{aligned}
$$

This completes the proof.

REMARK 3.2. If we apply well-known impulsive integral inequality of Gronwall-type in [4, Theorem 1.5.1.] to (3.6), then we also obtain the following estimate:

$$
\begin{aligned}
& |x(t)| \leq c\left|x_{0}\right| \varphi(t) \psi\left(t_{0}^{+}\right) \prod_{t_{0}<t_{k}<t}\left(1+c \rho_{k}\right) \exp \left[\int_{t_{0}}^{t} c \varphi(s) \psi(s) b(s) d s\right] \\
& =c\left|x_{0}\right| \varphi(t) \psi\left(t_{0}^{+}\right) \prod_{t_{0}<t_{k}<t}\left(1+c \rho_{k}\right) \exp \left[\int_{t_{0}}^{t} c b(s) d s\right], t \geq t_{0}
\end{aligned}
$$

\section{References}

[1] D. D. Bainov and P. S. Simeonov, Systems with Impulsive Effect: Stability, Theory and Applications, Ellis Horwood Series in Mathematics and Its Application, 1989.

[2] D. D. Bainov and P. S. Simeonov, Impulsive Differential Equations: Asymptotic Propertites of the Solutions, World Scientific Publishing Co., Inc., River Edge, NJ, 1995.

[3] S. K. Choi, N. Koo, and C. Ryu, Stability of linear impulsive differential equations via $t_{\infty}$-similarity, Chungcheong Math. Soc. 26 (2013), 811-819. 
[4] V. Lakshmikantham, D. D. Bainov, and P. S. Simeonov, Theory of Impulsive Differential Equations, World Scientific Publishing Co. Pte. Ltd., Singapore, 1989.

[5] V. Lakshmikantham, S. Leela, and A. A. Martynyuk, Stability Analysis of Nonlinear Systems, Marcel Dekker, INC., New York and Basel, 1989.

Department of Mathematics

Chungnam National University

Daejeon 305-764, Republic of Korea

E-mail: sgchoi@cnu.ac.kr

$* *$

Department of Mathematics

Chungnam National University

Daejeon 305-764, Republic of Korea

E-mail: njkoo@cnu.ac.kr

$* * *$

Department of Mathematics

Chungnam National University

Daejeon 305-764, Republic of Korea

E-mail: chmiry@hanmail.net 